\section{Exploring a 'pro-senescence' approach for prostate cancer therapy by targeting PTEN}

\section{Manuel Collado}

Spanish National Cancer Research Center (CNIO), 3 Melchor Fernandez Almagro Street, Madrid E-28029, Spain = Tel.: +34917328000 = Fax: +34917328033 = mcollado@cnio.es

Evaluation of: Alimonti A, Nardella C, Chen Z et al.: A novel type of cellular senescence that can be enhanced in mouse models and human tumor xenografts to suppress prostate tumorigenesis. J. Clin. Invest. 120(3), 681-693 (2010). Cellular senescence has proven its power in controlling tumor growth in various mouse models of cancer, and there are some hints pointing to its relevance in cancer chemotherapy. This article characterizes the induction of cellular senescence after decreasing the levels of tumor suppressor PTEN, and further investigates the potential therapeutic benefit of targeting cellular senescence induction for cancer therapy.

\section{Summary of methods \& results}

The stable cell growth arrest implemented by cells confronted by aberrant oncogenic signaling has been recognized as an efficient tumor-suppression mechanism opposing tumorigenesis in a number of tissues of mouse and human origin. Given the limited number of therapeutic approaches currently at our disposal in the fight against cancer, it would be wise to explore the usefulness of a pro-senescence alternative for cancer therapy.

Excessive oncogenic signaling emanating from the PI3K/AKT pathway, such as the one produced after deletion of the PTEN phosphatase, a negative regulator of the pathway and a crucial tumor suppressor in the prostate, triggers a form of cellular senescence that the authors termed PTEN-loss-induced cellular senescence (PICS) [1]. The detailed characterization of PICS revealed this cell growth arrest to have particular features that are different from those observed for oncogene-induced senescence (OIS), in that it proceeded in the absence of DNA replication and cellular proliferation and the ensuing DNA damage that typically accompanies OIS. These attributes make PICS an interesting candidate for cancer treatment. Genetic ablation, shRNA-mediated knockdown or pharmacological inhibition of PTEN (attained using a newly developed antidiabetic drug, VO-OHpic), all elicited a fast senescence response. Senescence induction was PTEN gene-dosage dependent, with no effect on wild-type cells, an increasing response in heterozygous to hypomorphic cells and with no further increase in completely nullizygous cells. The same effect was seen in human prostate cancer cells in vitro and, importantly, also in vivo. Cancer cells stopped proliferating and senesced in a xenograft model after systemic administration of VO-OHpic, only when the cells retained one copy of the PTEN gene and were wild-type $p 53$. These results led the authors to propose that the use of PTEN inhibitors to treat certain cancers might prove to be effective by inducing the tumor suppressive activity of cellular senescence.

Further characterization of the signaling pathways involved in PICS revealed a crucial role for the mTOR pathway, downstream of PTEN deletion. This was exposed by using rapamycin, a well-known immune-suppressive antifungal macrolide and inhibitor of the mTOR kinase complex 1 , and genetically by generating doubly deficient $P T E N^{-1-} m T O R^{-/-}$ mice. Surprisingly, the activity of mTOR that is relevant for PICS is the upregulation of $\mathrm{p} 53$, by controlling its rate of protein translation, and this activity seems to be dominant over the classical INK4A tumor suppressor gene product p19ARF-mediated p53 stabilization, which has been extensively reported as crucial in OIS. Accordingly, co-deletion of PTEN and $A R F$ did not block PICS or p53 upregulation and, again, these were rapamycin sensitive and, thus, mTOR dependent. These results led the authors to hypothesize that, since ARF does not oppose MDM2 to stabilize p53 during PICS, pharmacological inhibition of MDM2 with drugs such as Nutlin-3 would lead to maximal $p 53$ induction and potentiation of PICS. Indeed, treatment of mouse embryonic fibroblasts with Nutlin-3 after deletion of PTEN caused an enhancement of the senescent
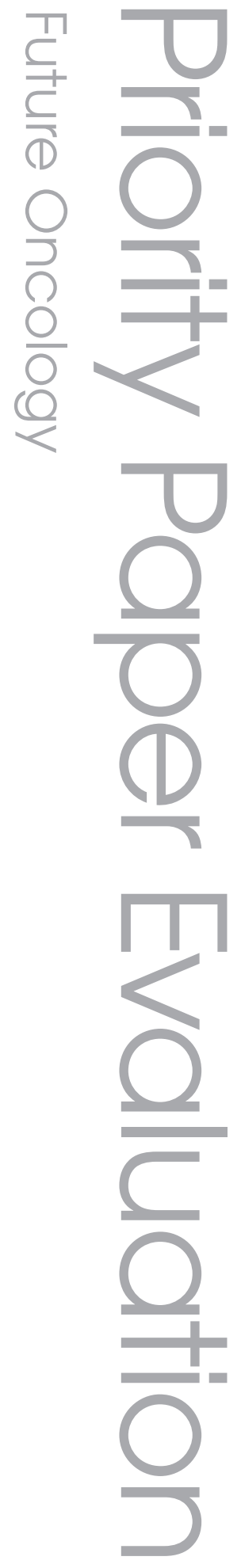

\section{Keywords}

- cancer therapy

- pro-senescence therapy

n PTEN = senescence

- tumor suppression

\section{future}


response. Interestingly, while rapamycin precluded the induction of senescence (in agreement with their previous results), treatment with Nutlin-3 after rapamycin restored the senescence response and cell growth arrest was maximal, showing that Nutlin-3 was dominant over rapamycin.

These results raised the possibility of enhancing PICS in vivo for tumor control with Nutlin-3 and provided support for a combinatorial therapy by using Nutlin-3 plus mTOR inhibitors to take advantage of the powerful cell growth-inhibitory action of the drug combination. To test this hypothesis, the authors assessed, in vivo, through a mouse model of PTEN-deletion-initiated prostate tumor, whether Nutlin-3 would control tumorigenesis by enhancing cellular senescence and whether this action can be potentiated by combining the treatment with RAD001, a well-known analog of rapamycin. Administration of Nutlin-3 to mice with prostate-specific deletion of PTEN prior to tumor onset reduced both tumor size and the number of affected glands per prostate. In line with all previous data reported on this study, the reduction in tumor burden was associated with an upregulation of $p 53$ and a clear senescence response. By contrast, RAD001, although resulting in tumor growth inhibition, prevented the upregulation of $p 53$ and decreased the senescent response. Remarkably, however, the combination of Nutlin-3 and RAD001 resulted in a dramatic reduction of tumor growth with a demonstrable enhanced senescence induction, validating in vivo the observed dominance of Nutlin-3-induced senescence over mTOR inhibition-mediated block in senescence and the synergistic effect on PICS achieved by combining both strategies.

\section{Discussion}

The PTEN protein is a lipid phosphatase that dampens down the proliferative and prosurvival activities of the PI3K/AKT signaling pathway. Increased PI3K/AKT activity is potentially protumorigenic and has been identified in a number of human tumors. One of the mechanisms by which tumor cells achieve increased activity of PI3K/AKT is by targeting PTEN, a molecular event that has been frequently observed in human cancer, especially in prostate cancer. However, partial retention of PTEN is extremely frequent in human cancer and particularly in prostate cancer, where wild-type $p 53$ can also be found. The authors previously demonstrated that deletion of PTEN induces senescence [2], and have now decided to further characterize this process and test the feasibility of taking advantage of its tumor-suppressive activity for cancer therapy. Detailed characterization of PICS showed that the process, in contrast to OIS, proceeds in the absence of proliferation and without a relevant DNA damage response. The authors claim that this offers advantages over OIS to target quiescent cells of the cancer stem cell type that would otherwise escape traditional cytotoxic drugs or a hypothetical OIS-based therapy that relies on proliferative cells. However, it should also be noted that inhibiting PTEN, a wellknown tumor suppressor, in normal cells might have detrimental consequences.

The authors went further to dissect the signaling downstream of PTEN loss and identified an mTOR-mediated increase in p53 translation as the relevant pathway triggering the cell growth arrest. Since ARF does not seem to be involved in the upregulation of $\mathrm{p} 53$, the authors tested the possibility of further enhancing PICS by blocking MDM2 using Nutlin-3. This proved to be the case, and administration of Nutlin-3 to a mouse model of PTEN-lossdriven prostate tumorigenesis was effective in reducing tumor progression by inducing cellular senescence. Furthermore, the combination of Nutlin-3 and mTOR inhibition, by targeting different activities downstream of PTEN, resulted in a more robust induction of cellular senescence and reduced tumor growth.

\section{Future perspective}

Originally considered the limit to the proliferative capacity of the cell [3], cellular senescence has proved to be a relevant and efficient tumor suppressor mechanism in mouse models and in human cancer [4]. Thus, it remains a promising therapeutic opportunity for the development of targeted anticancer therapies [5]. In this study, the authors characterized a particular form of cellular senescence and aimed to test the possibility of enhancing this response in vivo for tumor growth control.

Taken together, the results of this study support the feasibility of a pro-senescence therapy of cancer. More effective and specific anticancer treatments are highly demanded, and thus, novel strategies for the rational design of therapeutic interventions should provide us with new drugs to add to the weaponry at our disposal in the fight against cancer. Furthermore, they open up the interesting prospect of attacking 
tumors from different flanks, raising the possibility of enhancing the efficacy of current treatments by complementing and synergizing with them. This study is an excellent example of how the analysis of the molecular players involved in a particular form of cellular senescence can be used to exploit the knowledge gathered in vitro and in vivo with mouse models and human tumor xenografts to develop new therapeutic strategies to combat cancer.

\section{Financial \& competing interests disclosure}

The author has no relevant affiliations or financial involvement with any organization or entity with a financial interest in or financial conflict with the subject matter or materials discussed in the manuscript. This includes employment, consultancies, honoraria, stock ownership or options, expert testimony, grants or patents received or pending, or royalties.

No writing assistance was utilized in the production of this manuscript.

\section{Executive summary}

\section{Study design}

- Cellular senescence has been proven to have powerful anticancer potential in mouse models and some human clinical settings.

- Molecular characterization of cellular senescence might yield interesting new opportunities in the treatment of cancer.

\section{Results}

- Lowering the levels of tumor suppressor PTEN results in cellular senescence in the absence of proliferation or DNA damage, making it an attractive therapeutic intervention.

- Detailed molecular analysis of this type of senescence lent support for a potential anticancer activity, maximized in combination with drugs targeting p53 inhibitor MDM2, such as Nutlin-3a, and immunosuppressive drugs targeting mTOR, such as rapamycin or its analog RAD001.

- An in vivo experimental prostate cancer model demonstrated the efficacy of a pro-senescence anticancer therapy based on downregulation of PTEN.

\section{Conclusion}

- Drugs aimed at inducing cellular senescence represent a promising novel approach for cancer intervention.

\section{Bibliography}

1. Alimonti A, Nardella C, Chen Z et al:: A novel type of cellular senescence that can be enhanced in mouse models and human tumor xenografts to suppress prostate tumorigenesis. J. Clin. Invest. 120(3), 681-693 (2010).
2. Chen Z, Trotman LC, Shaffer D et al.: Crucial role of p53-dependent cellular senescence in suppression of PTEN-deficient tumorigenesis. Nature 436(7051), 725-730 (2005).

3. Collado M, Blasco MA, Serrano M: Cellular senescence in cancer and aging. Cell 130(2), 223-233 (2007).
4. Collado M, Serrano M: Senescence in tumours: evidence from mice and humans. Nat. Rev. Cancer 10(1), 51-57 (2010).

5. Roninson IB: Tumor cell senescence in cancer treatment. Cancer Res. 63(11), 2705-2715 (2003). 